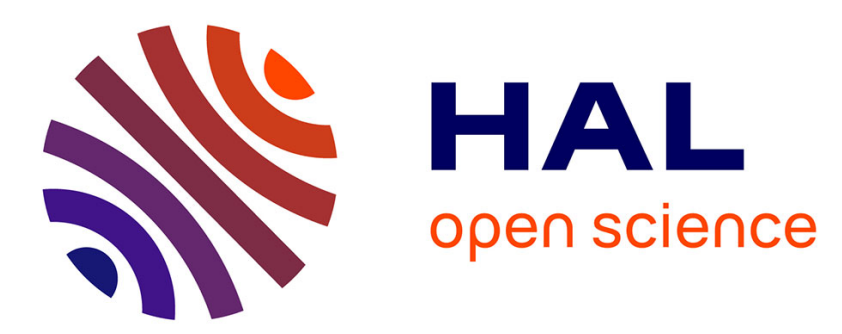

\title{
Decrease of intensity of ELF/VLF waves observed in the upper ionosphere close to earthquakes: A statistical study
}

F. Němec, O. Santolík, Michel Parrot

\section{- To cite this version:}

F. Němec, O. Santolík, Michel Parrot. Decrease of intensity of ELF/VLF waves observed in the upper ionosphere close to earthquakes: A statistical study. Journal of Geophysical Research Space Physics, 2009, 114 (A4), pp.n/a-n/a. 10.1029/2008JA013972 . insu-03037208

\section{HAL Id: insu-03037208}

\section{https://hal-insu.archives-ouvertes.fr/insu-03037208}

Submitted on 3 Dec 2020

HAL is a multi-disciplinary open access archive for the deposit and dissemination of scientific research documents, whether they are published or not. The documents may come from teaching and research institutions in France or abroad, or from public or private research centers.
L'archive ouverte pluridisciplinaire HAL, est destinée au dépôt et à la diffusion de documents scientifiques de niveau recherche, publiés ou non, émanant des établissements d'enseignement et de recherche français ou étrangers, des laboratoires publics ou privés. 


\title{
Decrease of intensity of ELF/VLF waves observed in the upper ionosphere close to earthquakes: A statistical study
}

\author{
F. Němec, ${ }^{1,2,3}$ O. Santolík, ${ }^{3,2}$ and M. Parrot ${ }^{1}$ \\ Received 8 December 2008; revised 1 February 2009; accepted 9 February 2009; published 2 April 2009.
}

[1] We present results of a systematic study of intensity of VLF electromagnetic waves observed by the DEMETER spacecraft in the upper ionosphere (altitude $700 \mathrm{~km}$ ). We focus on the detailed analysis of the previously reported decrease of wave intensity shortly before the main shock during the nighttime. Using a larger set of data (more than 3.5 years of measurements) and a newly developed data processing method, we confirm the existence of a very small but statistically significant decrease of wave intensity $0-4$ hours before the time of the main shock at frequencies of about $1.7 \mathrm{kHz}$. It is shown that the decrease does not occur directly above the earthquake epicenter but is shifted about $2^{\circ}$ in the westward direction. Moreover, it is demonstrated that the decrease occurs more often close to shallower earthquakes and close to earthquakes with larger magnitudes, as it is "intuitively" expected, representing an additional proof of validity of the obtained results. Finally, no dependence has been found on the occurrence of the earthquake below the ocean or below the continents.

Citation: Němec, F., O. Santolík, and M. Parrot (2009), Decrease of intensity of ELF/VLF waves observed in the upper ionosphere close to earthquakes: A statistical study, J. Geophys. Res., 114, A04303, doi:10.1029/2008JA013972.

\section{Introduction}

[2] The idea of additional electromagnetic phenomena accompanying earthquakes is rather old [Milne, 1890]. These might be of a large importance, because some of them are claimed to occur shortly (up to several days) before the time of the main shock and could therefore potentially serve as short-time precursors. Among other reported precursors (changes in temperature and concentration, resistivity changes, etc.), electromagnetic perturbations possibly connected with seismic activity have been recently discussed by several authors, both using ground-based [Tate and Daily, 1989; Asada et al., 2001; Bortnik et al., 2008] and satellite data [Parrot and Mogilevsky, 1989; Larkina et al., 1989; Molchanov et al., 1993, 2006; Parrot, 1994; Hobara et al., 2005; Němec et al., 2008]. The reported electromagnetic effects span over the large range of frequencies (from DC up to visible light), timescales (from several minutes up to a few months) and may be of various nature (enhancement of wave intensity, attenuation of wave intensity, modification of wave characteristics). On the other hand, there is a number of studies that reveal no or only a very weak correlation between the seismic activity and observed effects [Henderson et al., 1993; Rodger et al.,

\footnotetext{
${ }^{1}$ Laboratoire de Physique et Chimie de l'Environnement et de l'Espace, Centre National de la Recherche Scientifique, Orléans, France.

${ }^{2}$ Faculty of Mathematics and Physics, Charles University, Prague, Czech Republic.

${ }^{3}$ Institute of Atmospheric Physics, Academy of Sciences of the Czech Republic, Prague, Czech Republic.
}

Copyright 2009 by the American Geophysical Union. 0148-0227/09/2008JA013972
1996; Clilverd et al., 1999]. These negative results might possibly be due to the fact that any potential precursors are very weak and could be therefore easily hidden in the common variations of the natural background. In addition, it is quite complicated to compare the different performed studies, because they often use very different data set and data processing methods. Finally, theoretical models that have been developed in order to explain the precursory phenomena are numerous [Gershenzon et al., 1989; Molchanov et al., 1995, 2001; Molchanov and Hayakawa, 1998; Sorokin et al., 2001; Pulinets et al., 2003; Freund, 2007], but they mostly represent only physical ideas. The mechanisms that are responsible for potential earthquake precursors are still understood very poorly.

[3] The presented paper closely follows a study by Němec et al. [2008], where we systematically investigated a large set of satellite data (more than 2.5 years) and showed that during the night there is a small but statistically significant decrease of wave intensity in the vicinity of large shallow earthquakes shortly ( $0-4$ hours) before the time of the main shock. The observed effect was strongest for earthquakes with magnitudes larger than or equal to 5.0 and depths lower than $40 \mathrm{~km}$. No effect was observed during the day nor for deep earthquakes.

[4] In the first part of the present paper we analyze a larger data set (more than 3.5 years) by using the same data processing method as formerly used by Němec et al. [2008]. Then, we apply a newly developed data processing and study the observed effect more in detail, focusing namely on its variation with different earthquake parameters: magnitude, depth, altitude of the solid surface above the hypocenter (whether the earthquake occurred below the ocean or below the continent). Data set that we have used for the 


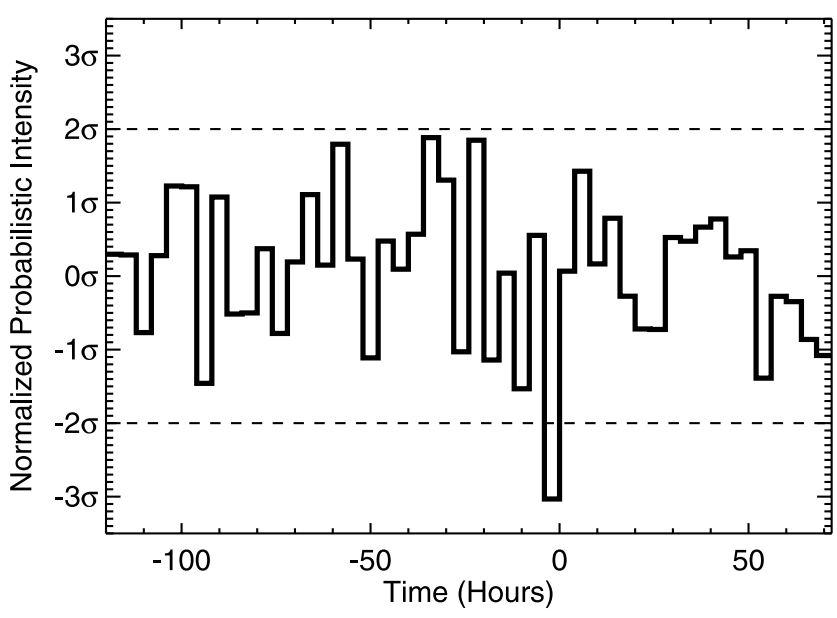

Figure 1. Normalized probabilistic intensity as a function of the time relative to the time of the main shock (see text).

study is presented in section 2 . Section 3 presents results of the data processing method introduced by Němec et al. [2008] applied on the substantially extended data set. The newly developed data processing method is described in section 4, whereas section 5 presents the obtained results. These are discussed in section 6 and summarized in section 7.

\section{Data Set}

[5] For this study, data from the French spacecraft DEMETER have been used. DEMETER is a microsatellite launched in June 2004 on a circular quasi sun-synchronous orbit (10.30 and $22.30 \mathrm{LT}$ ) with an altitude of about $700 \mathrm{~km}$ [Parrot, 2006] and inclination $98^{\circ}$. The altitude was changed to about $660 \mathrm{~km}$ in December, 2005. The satellite performs 14 orbits per day and measures continuously between $-65^{\circ}$ and $+65^{\circ}$ of geomagnetic latitude, providing thus a very good coverage of the Earth's seismic zones. Among several instruments placed on board, we have focused on the analysis of electric field data measured by the ICE instrument [Berthelier et al., 2006]. Irrespective of the mode, this provides us with on-board calculated power spectrum of one electric field component. The frequency resolution is $19.5 \mathrm{~Hz}$ and time resolution is $2 \mathrm{~s}$ or $0.5 \mathrm{~s}$ depending on the mode of operation. For the data set used in this study the selected electric component is perpendicular to the orbit plane. Altogether, we have used data from more than 3.5 years of the satellite measurements, representing about 9000 hours of nighttime data in about 15500 orbits. During the analyzed period about 9500 large shallow earthquakes with magnitude larger than or equal to 4.8 and depth less than $40 \mathrm{~km}$ occurred all over the world according to the USGS catalog (http://neic.usgs.gov/neis/epic/epic global.html). Among these, there are more than 5500 earthquakes with magnitude larger than or equal to 5.0.

\section{Verification of Results of Němec et al. [2008] Using a Larger Data Set}

[6] We have used a larger data set (46 months as compared to 30 months of Némec et al. [2008]) and verified the existence of the previously reported effect [Němec et al., 2008]. In order to enable a direct comparison with the formerly obtained results, we have used exactly the same data processing method (see Appendices A and B for a detailed description). We have focused on a specific frequency-time interval and a range of earthquake parameters for which the effect has been found; we have analyzed only the nighttime data and the $200 \mathrm{~Hz}$ wide frequency band centered at $1.7 \mathrm{kHz}$. Moreover, we have limited our study only to earthquakes with magnitudes larger than or equal to 5.0 and with depths shallower than $40 \mathrm{~km}$.

[7] The results that we have obtained for distances less than $3^{\circ}$ from the epicenters of earthquakes are represented in Figure 1. It shows the normalized probabilistic intensity as a function of the time relative to the time of the main shock, spanning from 5 days before to 3 days after. The time resolution is 4 hours. It can be seen that the normalized probabilistic intensity, normally fluctuating between $-2 \sigma$ and $2 \sigma$ ( $\sigma$ being the standard deviation), decreases shortly before the time of the main shock to less than $-3 \sigma$. There are about 70 events included in each of the bins. The decrease occurs $0-4$ hours before the time of the main shock and is formed by 2068 points coming from 64 different events. The mean value of probabilistic intensity in this bin (see Appendix B) is -0.083 , corresponding to a decrease of wave intensity equal to about $-2.4 \mathrm{~dB}$. The median value of probabilistic intensity in this bin is -0.126 , corresponding to a decrease of wave intensity equal to about $-3.6 \mathrm{~dB}$. Although these values represent a small but significant decrease of wave intensity as compared to the normal values, the absolute value of this decrease is somewhat smaller than the one reported by Němec et al. [2008] (see a detailed discussion in section 6).

[8] A distribution of all the cumulative probabilities that contribute to the bin where a decrease of wave intensity is observed is shown by a solid line in Figure 2. The mean/ median value is marked by dotted/dashed line, respectively. If there were no effects connected with the seismic activity, the distribution should be approximately uniform (see Appendix A), which is not the case; lower values of cumulative probability clearly occur more often, corresponding to a decrease of wave intensity. For comparison, we show by a dash-dotted line a distribution of cumulative probabilities in all bins but the one where the effect is observed.

\section{New Data Processing Method}

[9] We have developed a new data processing method designed specifically to check for the presence of seismicrelated effects in a given frequency and time (relative to the time of the main shock) intervals. Following the conclusions of Němec et al. [2008], we have focused solely on the analysis of the nighttime data, $200 \mathrm{~Hz}$ wide frequency band centered at $1.7 \mathrm{kHz}$ and time interval $0-4$ hours before the time of the main shock. These are exactly the same parameters, for which a decrease of wave intensity has been previously reported.

[10] The applied data processing can be divided into 4 steps, among which the first two are analogical to those previously introduced by Němec et al. [2008]; see Appendix A.

[11] The third level of data processing is different: we select a point $P$ within $\pm 10^{\circ}$ in latitude and $\pm 10^{\circ}$ in longitude from the earthquake epicenter. For each of the orbits that enters a circle with a radius of $3^{\circ}$ from the point $P$ 


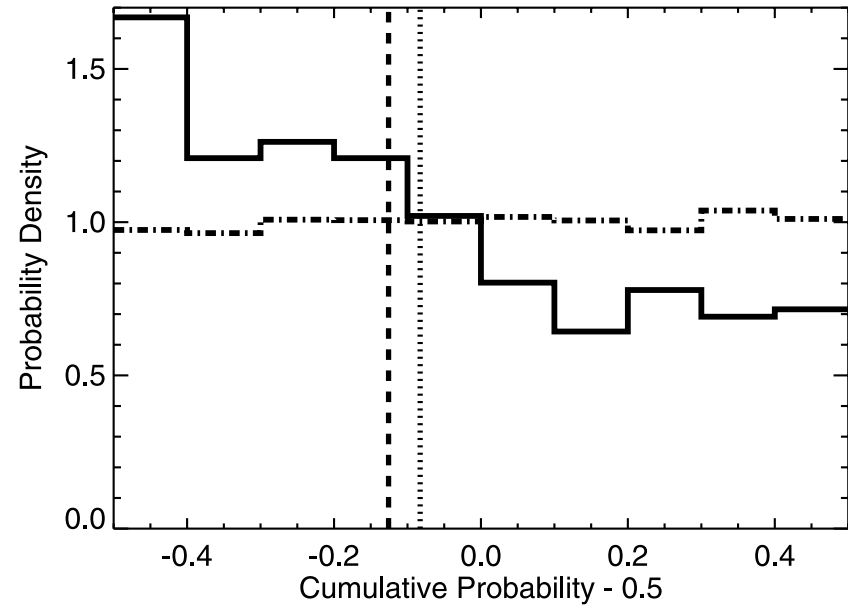

Figure 2. (solid line) Distribution of all the cumulative probabilities that contribute to the observed decrease of wave intensity. Mean/median value is plotted by a dotted/ dashed line, respectively. (dash-dotted line) Distribution of cumulative probabilities in all bins, but the one wherein the effect is observed.

we check whether the values of the cumulative probability (that means the "normalized" intensities) are lower than or larger than normal ones close to the point. In order to do this, we take advantage of the Mann-Whitney $U$ test [Sheskin, 2000]. This is a statistical test that enables, on a given level of significance, a check to see if two populations $X$ and $Y$ have the same mean of distribution or not. The reason for using the Mann-Whitney $U$ test instead of the more traditional Student $t$ test [Sheskin, 2000] is that we deal with non-Gaussian distributions. This does not represent a problem for the Mann-Whitney $U$ test, which is a nonparametric test. As a population $X$ we take the cumulative probabilities close to the point $P$ and as a population $Y$ we take the cumulative probabilities in the rest of the same half-orbit. The reason for this treatment is that each halforbit lasts for only about 35 minutes, which is a relatively short time as compared to the timescale of major changes of intensity of electromagnetic waves in the upper ionosphere. The values measured all over the half-orbit are therefore dependent and it is reasonable to take as normal "background" values the values of cumulative probabilities in the rest of a given half-orbit.

[12] The fourth step consists of a simple statistical evaluation of the obtained results. For each of the events (that is for each of the orbits that encounters the point $P$ sufficiently close at the given time) and a predefined level of significance there are three possibilities: (1) cumulative probabilities close to the point $P$ are lower than in the rest of the half-orbit, (2) cumulative probabilities close to the point $P$ are larger than in the rest of the half-orbit, and (3) cumulative probabilities are about the same close to the point $P$ and in the rest of half-orbit; at a predefined level of significance it is not possible to decide which of them are lower.

[13] The events for which it is not possible to draw a clear conclusion using the Mann-Whitney test are not taken into account in the further data processing. We calculate the number of events for which the values of cumulative probabilities close to the point $P$ are lower than in the rest of the half-orbit $\left(N_{d}\right)$ and, in the same way, we calculate the number of events for which the values of cumulative probabilities close to the point $P$ are larger than in the rest of the half-orbit $\left(N_{i}\right)$. Directly from these two values we can calculate the probability of their random occurrence. If we suppose no effect connected with the seismic activity, the probability $p_{i}$ of an increase of intensity close to the point $P$ would be the same as the probability $p_{d}$ of a decrease of intensity close to the point $P$ and the numbers $N_{i}$ and $N_{d}$ would be consequently about the same. If the numbers $N_{i}$ and $N_{d}$ are significantly different, it means that the wave intensity is different close to the point $P$ than in the rest of the half-orbits, indicating the existence of a seismic-related effect. The subsequent evaluation of the probability of occurrence is very trivial: since $p_{i}=p_{d}$ for an unperturbed case, all that we have to deal with is a simple binomial distribution.

\section{Results}

[14] A left panel of Figure 3 represents a schematic view of the geometry used for construction of latitude-longitude plot. There is an epicenter of the earthquake in the middle of Figure 3 and longitudinal/latitudinal distances from it are plotted on the horizontal and vertical axis, respectively. A dashed line represents a part of the satellite orbit and a cross in the upper right part of Figure 3 represents an arbitrary example point: we want to check whether the emissions in its vicinity have unusually large or low intensity. Consequently, we simply compare the cumulative probabilities measured close to the point (closer than $3^{\circ}$, following Némec et al. [2008], inner part of the marked circle) with the cumulative probabilities measured during the same half-orbit farther than $3^{\circ}$ from the point using the MannWhitney test and use the data processing described above in section 4.

[15] The right panel of Figure 3 shows the results obtained for the latitude-longitude plot. Only sufficiently large (magnitude $\geq 5.0$ ) and shallow (depth $<40 \mathrm{~km}$ ) earthquakes have been used for its construction. The longitudinal and latitudinal distances from earthquakes are plotted on the horizontal and vertical axis, respectively. The color scale represents the probability of random occurrence. It can be seen that the only exceptional effect is located close to the epicenter of earthquakes, shifted by about $2^{\circ}$ in the Westward direction and also slightly to the North. It represents a decrease of the wave intensity. Note that this cannot be determined from the plotted color scale, because it represents only a probability that such values $N_{d}$ and $N_{i}$ could occur randomly, not which of them is larger. We have used the significance level for the Mann-Whitney test equal to 0.01 , leading to about 50 events per each bin of the resulting plot. However, the results are independent of this threshold (see section 6 for more discussion).

[16] Having observed a decrease of the wave intensity close to the earthquakes, we have focused on its further analysis. More specifically, we were interested how it depends on various parameters. In order to do so, we have compared the data in a $3^{\circ}$ radius around the point located $2^{\circ}$ to the West from the epicenter (that is approximately the area where the decrease is observed) and the rests of the 

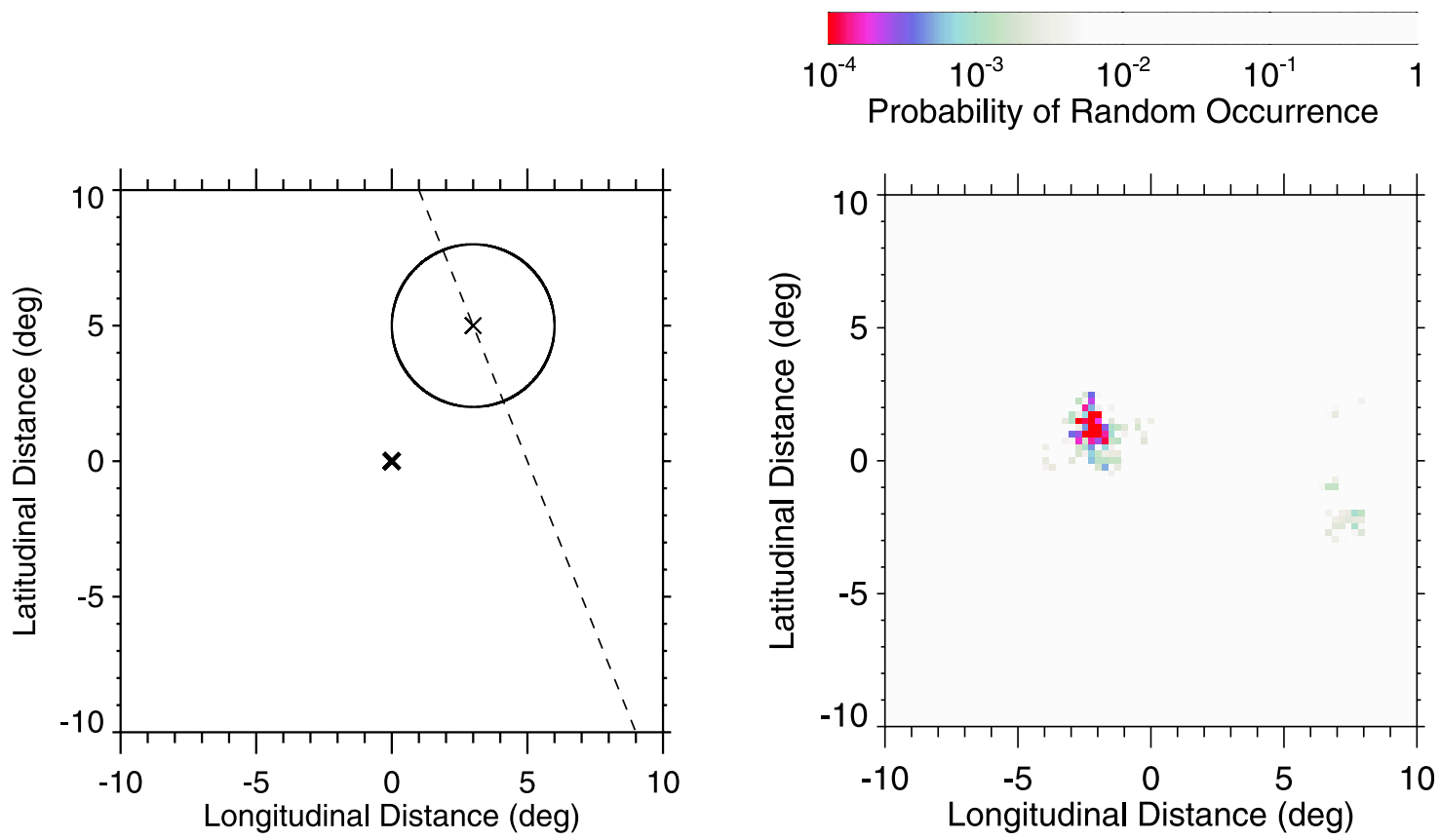

Figure 3. (left) Geometry of latitude-longitude plot: scheme of calculation. (right) Latitude-longitude plot of probability of random occurrence of such a ratio of decreases/increases for earthquakes with magnitudes of $\geq 5.0$ and depths of $<40 \mathrm{~km}$.

half-orbits, using the data processing described in section 4 . Afterward, we have checked how $N_{d}$ and $N_{i}$ depend on the magnitude of the earthquake, depth of the earthquake and altitude of the solid surface above the hypocenter (whether the earthquake occurred below the ocean or below the continent). The level of significance used for the MannWhitney test was again 0.01 , as it is throughout the present paper.

[17] The results that we have obtained for the dependence on the magnitude of the earthquakes are shown in Figure 4. In Figure 4 we have slightly decreased the threshold of magnitude down to 4.8. The left panel represents $N_{i}$ as a function of the magnitude of the earthquakes, while the right panel represents the same dependence of $N_{d}$. All shallow (depth $<40 \mathrm{~km}$ ) earthquakes were taken into account when constructing the plot. As expected, weaker earthquakes occur more often and the wave intensity in their vicinity can both increase or decrease. We expect that for even lower magnitudes $(<4.8)$ the decreases and increases would occur with about the same probability. It can be seen that for sufficiently large earthquakes (magnitude $\geq 5.5$ ) there is almost always a decrease of wave intensity close to the earthquakes (15 events out of 17). Please note that the peak in the magnitude range 5.4-5.7 is most probably just a random fluctuation caused by a low number of events.

[18] Figure 5 is similar as Figure 4, but this time we have focused on the dependence of the observed effect on the depth of the earthquakes. All large (magnitude $\geq$ to 5.0) earthquakes were taken into account when constructing the plot. It can be seen that for very shallow earthquakes (depth $<20 \mathrm{~km}$ ), decrease of wave intensity close to earthquake occurs more often. Please note that the large number of included earthquakes with depths about $10 \mathrm{~km}$ is already contained in the used USGS catalog, which attributes this depth to a very large number of events.

[19] After having investigated a dependence on the magnitude (Figure 4) and depth (Figure 5) of the earthquake separately, we combined them into a single plot, which is shown in Figure 6. It represents color-coded relative number of decreases (that is $N_{d} /\left(N_{d}+N_{i}\right)$ ) as a function of magnitude (horizontal axis) and depth of the earthquake (vertical axis). Relative number of decreases equal to 0.5 means that the number of decreases of the wave intensity close to earthquakes is equal to the number of increases of the wave intensity close to earthquakes, meaning thus no specific change of wave intensity connected with the earthquakes. On the other hand, relative number of decreases equal to 1 means that for all of the analyzed events the cumulative probabilities were lower in the vicinity of earthquakes than in the rest of the half-orbits (or it was not possible to decide using the Mann-Whitney test at a given level of significance, see section 6 for a detailed description). The used significance level of the Mann-Whitney test equal to 0.01 resulted in about 10 events per each bin. It can be seen that while the relative number of decreases is close to 0.5 for deep earthquakes with low magnitude (top left corner), meaning no change of wave intensity close to the earthquakes, it is equal to 1 for shallow earthquakes with large magnitudes (bottom right part).

[20] Figure 7 represents a similar plot as Figures 4 and 5, but this time the results were obtained for dependence on the altitude of the solid surface above the hypocenters of the earthquakes (magnitude $\geq$ to 5.0 ). It can be seen that the number of earthquakes occurring under the ocean (altitude less than $0 \mathrm{~km}$ ) is much larger than the number of earthquakes occurring under the land (altitude larger than $0 \mathrm{~km}$ ). However, no clear dependence of the studied effect can be 

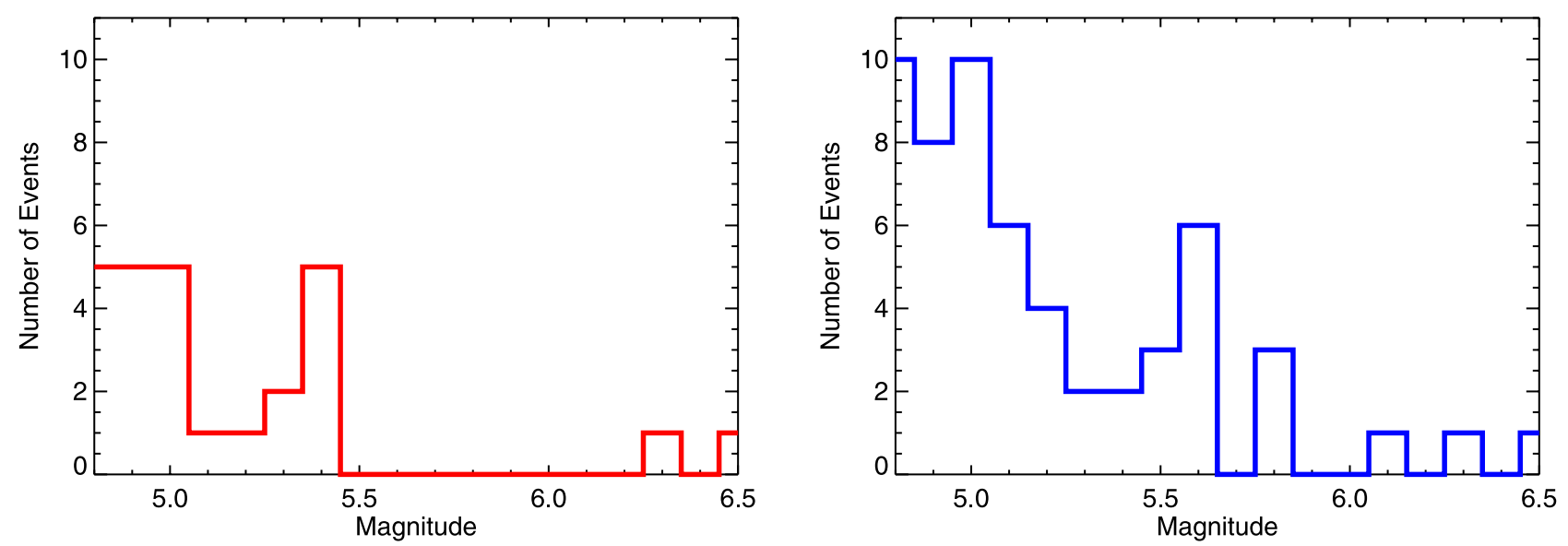

Figure 4. (left) Number of events with an increase of wave intensity close to the epicenter of earthquake $\left(N_{i}\right)$ as a function of magnitude of earthquake. (right) Number of events with a decrease of wave intensity close to the epicenter of earthquake $\left(N_{d}\right)$ as a function of magnitude of earthquake.

observed; the ratio of the number of wave intensity decreases to the number of wave intensity increases does not seem to depend on whether the earthquakes occur below the ocean or below the continent.

\section{Discussion}

[21] Data from more than 3.5 years of measurements performed by the DEMETER spacecraft and earthquakes occurring all over the world have been used. This represents a unique data set, ideal for performing large systematic studies. However, one must keep in mind a basic limitation of such kind of surveys: although a large number of earthquakes occurred during the analyzed period, there is only a small number of them which DEMETER encountered very close. This significantly complicates any statistical analysis of seismic-related effects, because it is reasonable to suppose the effects to be limited to some area around the epicenter of an earthquake. Consequently, although large volumes of data are measured, only a low number of measurements occur in the vicinity of large earthquakes and are potentially interesting. From this point of view the presented data processing that takes advantage of the map of

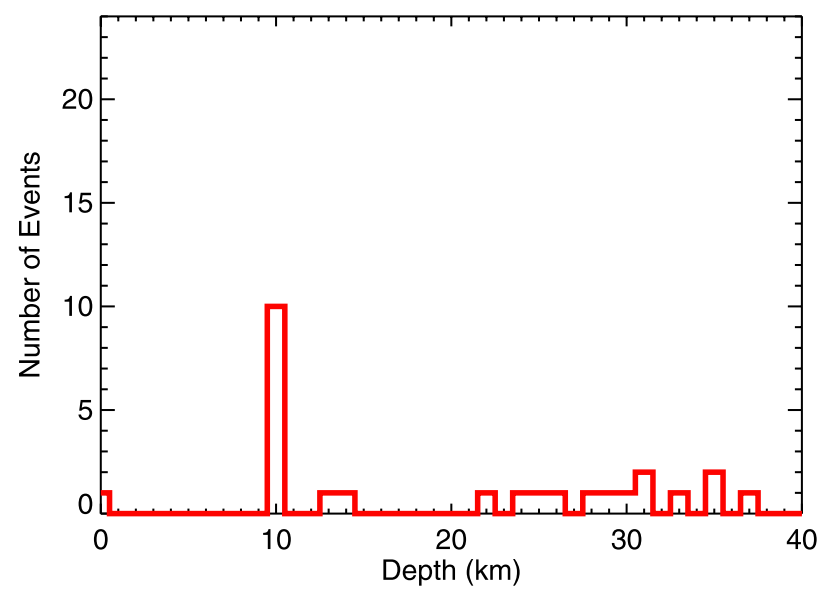

electromagnetic emissions is optimal, because it uses these far-from-earthquakes data at least in order to estimate normal (that is seismically unperturbed) values of the wave intensity.

[22] The previously reported decrease of the wave intensity shortly before the time of the main shock [Němec et al., 2008] has been confirmed when using a larger data set. However, the amplitude of the observed effect (mean/median value of $-0.083 /-0.126$, respectively, corresponding to about $-2.4 \mathrm{~dB} /-3.6 \mathrm{~dB}$, respectively) is weaker than the amplitude reported by Němec et al. [2008] (mean/median value of $-0.146 /-0.176$, corresponding to about $-4.2 \mathrm{~dB} /$ $-5.1 \mathrm{~dB}$, respectively) using a smaller set of data. A careful check of all the 64 events forming the decrease reveals that this is due to a few recent events with large probabilistic intensities, which significantly increase the resulting mean value of the probabilistic intensity.

[23] This represents a basic constrain of the statistical method introduced by Němec et al. [2008]: checking the mean value of cumulative probability is a good tool for revealing any systematic changes of wave intensity connected with seismic activity using a large data set. However, the effects due to the seismic activity are very

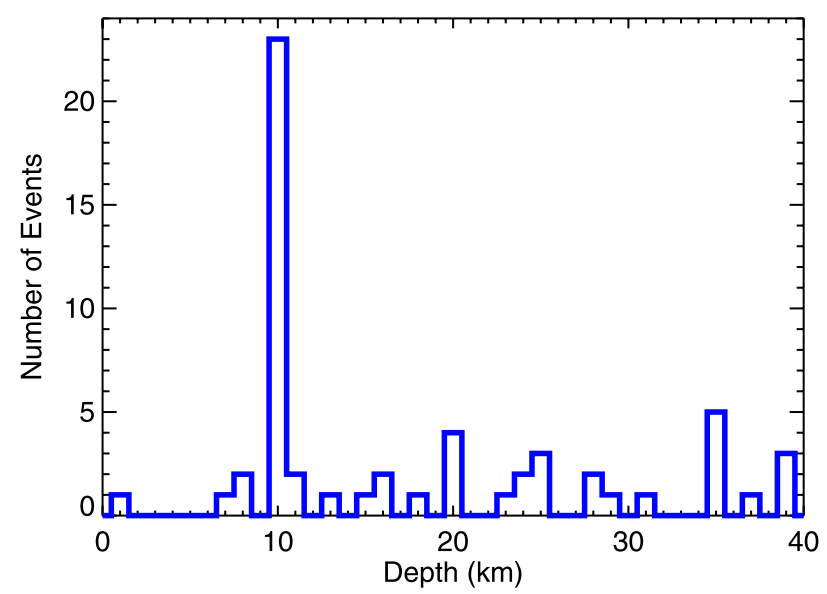

Figure 5. The same as Figure 4, but for the dependence on the depth of earthquakes. 


\section{$\begin{array}{llllll}0.5 & 0.6 & 0.7 & 0.8 & 0.9 & 1.0\end{array}$ Relative Number of Decreases}

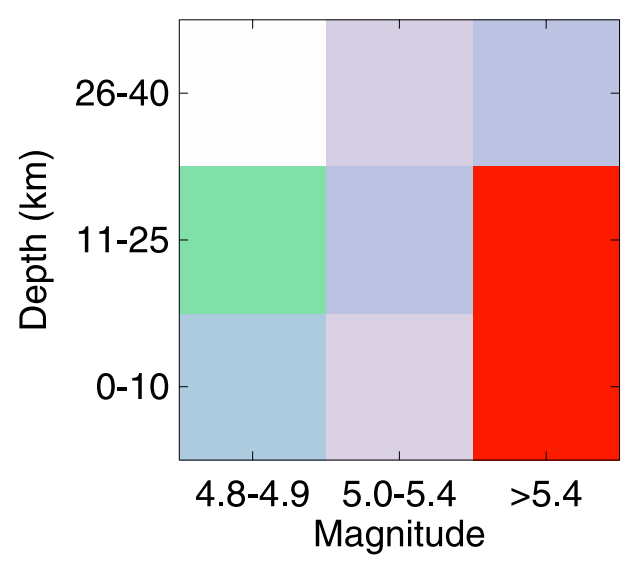

Figure 6. Relative number of decreases in the vicinity of earthquakes as a function of their depth and magnitude.

small as compared to the common natural variations of wave intensity. For an individual event (or a small number of events), the mean value of cumulative probability is therefore linked to these random natural variations rather than to seismic-related effects. A large value of the mean cumulative probability for a single event thus does not mean that there was an increase of wave intensity close to the earthquake, but more likely that the wave intensity during that time was globally larger than normal. This is a direct consequence of comparing close-quake data to the data measured at a given place under the similar conditions all over the analyzed period of several years. Surely, an inverse effect exists as well; low values of cumulative probability can be caused by the wave-quiet period and not by seismicrelated effects.

[24] From this analysis, we can only conclude that although the decrease of wave intensity is statistically significant (more than 3 standard deviations), but very small

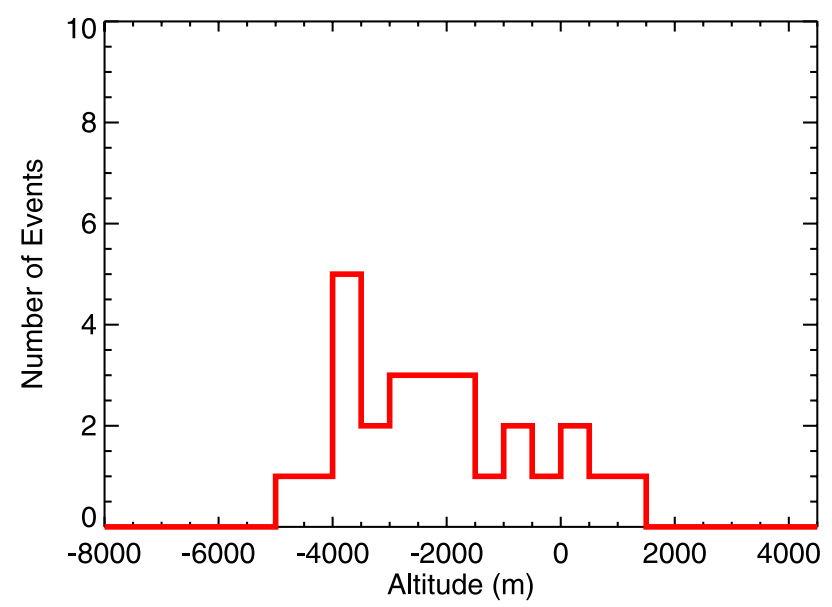

(about $3 \mathrm{~dB}$ ) as compared to the usual natural variability (about $11 \mathrm{~dB}$ ).

[25] The newly developed data processing method takes advantage of the Mann-Whitney test at a specified level of significance. The obtained results thus necessarily depend on this choice. In order to remain consistent, we have used the value of 0.01 throughout the paper. However, it turns out (not shown) that as long as the choice of the level of significance remains "reasonable," the obtained results remain practically unchanged. If too low level of significance is required, than the test is able to provide a clear answer only for a small number of half-orbits and the resulting statistics is poor. On the other hand, if the level of significance is chosen to be too large, the test provides a result even if it is practically impossible to decide which cumulative probabilities are lower: many "unclear" events enter the statistics and the real effects are buried in a random noise.

[26] While in the former study the effect was found to occur within $3^{\circ}$ from the epicenter of an earthquake, a newly developed data processing enables a more detailed check of the position and shape of the affected area. It turns out that instead of occurring directly above the epicenter of an earthquake, the effect is shifted by about $2^{\circ}$ to the West and slightly to the North. The observed longitudinal shift might possibly be caused by the Earth's rotation and the resulting Coriolis force: if there are, for example, aerosols [Pulinets and Boyarchuk, 2004] propagating toward the larger altitudes, it would always be dragged to the west. Another possibility could be that the origin of the effect is somehow related to the ions, which drift in the westward direction because of the Earth's magnetic field. Concerning the northward drift, we do not have any explanation at this moment.

[27] A detailed analysis of the observed effect is very important for two reasons. First, a comprehension of properties of the observed phenomenon is crucial for understanding the physical mechanism which causes the effect. Second, and at the present level of research perhaps even more importantly, it can serve as an independent test of the validity of the obtained results. This is possible, because at least for some of the analyzed parameters we have a good

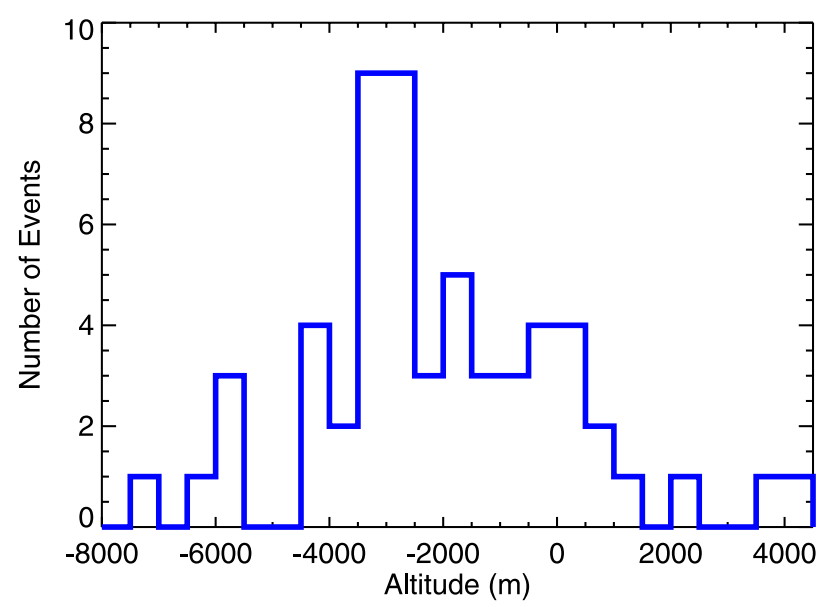

Figure 7. The same as Figure 4, but for the dependence on the altitude of solid surface above earthquake. 
"intuitive" idea of what the dependence should look like. For example, it is reasonable to expect that the observed effect would be stronger for larger earthquakes. Additionally, the effect should be stronger for shallower earthquakes: for these, any signal propagating from the region of a future earthquake does not have to pass that far through the lithospheric material before reaching the satellite altitude. Our results confirm both these "intuitive" expectations, which is very important.

[28] At this place we would like to shortly comment on the result shown in Figure 6. The reason is that although the relative number of wave intensity decreases is equal to 1 for large shallow earthquakes, it does not mean that the cumulative probabilities are always lower close to their epicenters than in the rest of the orbits for earthquakes with such parameters; it can also mean that in some cases there is no significant change of cumulative probabilities close to the epicenters as compared to the rests of the half-orbits. In such cases, the Mann-Whitney test cannot determine which set of values is lower and the events are not taken into account. For the used level of significance 0.01 , there is usually about $50 \%$ of such events. Consequently, we can only say that if the spacecraft passes close to the large shallow earthquake, there is either no change of cumulative probability or there is a decrease of cumulative probability. Moreover, it should be noted that the statistics for this plot is rather poor, there is only about 10 events in each of the bins. Unfortunately, this cannot be improved anyhow and represents a basic limitation of all the statistical studies of this type.

[29] The last dependence that we have checked was whether the observed effect depends on the altitude of the solid surface above the hypocenter of an earthquake or not. This could help with identification of physical mechanisms which are causing the phenomenon: some of the proposed mechanisms [Parrot, 1995] should work for earthquakes below the sea level as well (e.g., radon emanation) while others should work only for land earthquakes. Although at the present level of knowledge we do not dare to discuss about the possible mechanism, we believe that our finding that there is no clear dependence of the effect on the altitude of the solid surface above the hypocenter of an earthquake is very important for further theoretical development.

[30] Finally, we would like to underline that although the correlation between seismic activity and intensity of electromagnetic waves is statistically significant, it is observed only due to the large number of the analyzed events. Therefore even if there is on average a decrease of wave intensity related to large surface earthquakes, individual events may exhibit rather different behavior; the natural fluctuations of intensity of electromagnetic waves are large and the observed effect is relatively weak as compared to them.

\section{Conclusions}

[31] We have studied the previously reported decrease of the intensity of electromagnetic waves in the vicinity of earthquakes [Němec et al., 2008]. We have confirmed that during the night there is a very small but statistically significant decrease of wave intensity observed by the satellite close to large surface earthquakes shortly before the time of the main shock. Using a newly developed data processing method, we have shown that

[32] 1. the effect is stronger for larger earthquakes,

[33] 2. the effect is stronger for shallower earthquakes,

[34] 3. the effect does not seem to depend on whether the earthquake occurs below the ocean or not,

[35] 4. the effect occurs slightly $\left(\approx 2^{\circ}\right)$ to the west from the epicenter of earthquakes.

[36] The first two points are in a good agreement with what is "intuitively" expected. On the other hand, the last two points might be important for understanding the physical mechanism that is responsible for the effect.

\section{Appendix A: Detailed Description of the First Two Steps of the Data Processing, Based on Němec et al. [2008]}

[37] The purpose of Appendix A is to provide a detailed description of the first part of the data processing method. This part is common for both the method by Némec et al. [2008] and the newly developed method described in section 4.

[38] In the first step of the data processing, a map of electromagnetic emissions is constructed. This is built using all the measured data and can be represented by a sixdimensional matrix with the indices of the following meaning:

[39] 1. frequency (any frequency bands that we are interested in);

[40] 2. geomagnetic longitude of the satellite (longitudinal resolution $10^{\circ}$ );

[41] 3. geomagnetic latitude of the satellite (latitudinal resolution $2^{\circ}$ );

[42] 4. magnetic local time (daytime and nighttime; there are no other possibilities, as described in section 2);

[43] 5. magnetospheric conditions described by the $\mathrm{Kp}$ index: three bins made in such a way that there is about the same amount of data accumulated in each of the bins $(0-10$, $1+-2+$, above $3-$ );

[44] 6. season of the year (October-April, May-September).

[45] In each cell of this matrix we accumulate a histogram of the common logarithm of power spectral density of electric field fluctuations. Consequently, for a given location of the spacecraft (geomagnetic longitude and magnitude), magnetospheric conditions (magnetic local time, $\mathrm{Kp}$ index, season of the year) and frequency we obtain an estimate of the probability density function $f(E)$ of observing a power spectral density of electric field fluctuations $E$.

[46] In the second step of the data processing, we attribute a cumulative probability $F_{i}$ to each of the measured power spectral densities $E_{i}$. The appropriate value of the cumulative probability $F_{i}$ is equal to the value of cumulative distribution function obtained for the same frequency, spacecraft location and similar geomagnetic conditions. This can be calculated directly as an integral of the appropriate probability density function obtained in the first step of the data processing:

$$
F_{i}=\int_{-\infty}^{E_{1}} f(E) d E
$$

Therefore the cumulative probability $F_{i}$ is a number between 0 and 1 that represents the probability of 


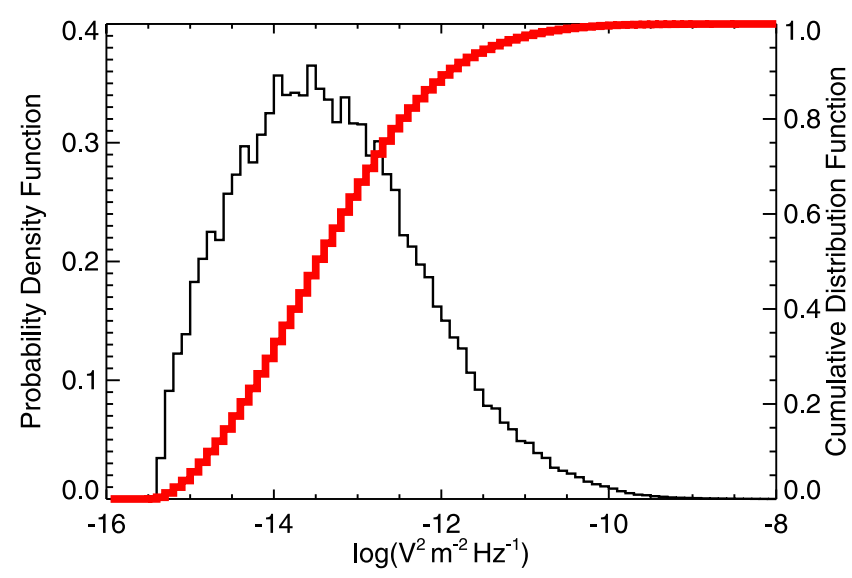

Figure A1. Probability density of observing a given power spectral density of electric field fluctuations (thin) and the corresponding cumulative distribution function (thick).

occurrence of signals with a power spectral density lower than or equal to the measured power spectral density of electric field fluctuations $E_{i}$. At this point, it is important to notice that the values of cumulative probability calculated from the entire data set (that is the same data set as has been used for construction of map of electromagnetic emissions) are uniformly distributed between 0 and 1 . This is a direct consequence of the definition of cumulative probability.

[47] Figure A1 represents an example of distribution of observed power spectral densities (thin) and also the corresponding cumulative distribution function (bold). The nighttime data in a frequency band centered at $1.7 \mathrm{kHz}$ measured all over the world during all the analyzed period have been used when constructing the plot. When performing the calculation, we have one such a plot for each combination of frequency band, spacecraft location, magnetic local time, geomagnetic conditions and season of the year, which enables a direct conversion between the power spectral density of electric field fluctuations and the corresponding value of the cumulative distribution function (cumulative probability).

\section{Appendix B: Detailed Description of Data Processing Using the Normalized Probabilistic Intensity Based on Němec et al. [2008]}

[48] The purpose of Appendix B is to provide a detailed description of the data processing method based on the normalized probabilistic intensity used by Němec et al. [2008].

[49] After applying the two steps described in Appendix A, data whose distance from the epicenter is lower than some threshold (a threshold of $10^{\circ}$ has been used) and which occur in the requested time interval (a time interval from 5 days before to 3 days after the time of the main shock has been chosen) are evaluated. If two or more earthquakes occur in the required distance/time interval from the measurement (and therefore possibly influence the data), the measurement is not taken into account. This condition, which is basically similar to taking into account only "individually occurring" earthquakes, is very important, because otherwise it could happen that a single measurement is attributed to more than one earthquake and used more than once. Having selected the proper data that occur close to earthquakes (both in time and space), the corresponding cumulative probabilities are calculated. These are then organized in bins as a function of the following parameters:

[50] 1. time to/from the time of the main shock (resolution of 4 hours),

[51] 2. distance from the epicenter of earthquake (resolution of $1^{\circ}$ ),

[52] 3. frequency (the same frequency bins as for the map of electromagnetic emissions).

[53] For each of the bins we define a "probabilistic intensity." For a bin $b$ it is calculated as follows:

$$
I_{b}=\frac{\sum_{i=1}^{M_{b}} F_{i}}{M_{b}}-0.5,
$$

where $M_{b}$ is the number of cumulative probabilities $F_{i}$ collected in a given bin. In other words, we calculate the mean value of cumulative probability in the bin and subtract one half in order to obtain a number between -0.5 and 0.5 instead of between 0 and 1 . Now if the observed intensities that belong to the bin were significantly lower/larger than the usual ones, the attributed cumulative probabilities would be significantly lower/larger than 0.5 and the resulting probabilistic intensity would be significantly lower/larger than 0 . The only remaining task is what quantitative value should be attributed to the word "significantly." This can be solved using statistical properties of the probabilistic intensity.

[54] The uniformly distributed values of probabilistic intensity are averaged into a bin $b$ corresponding to a given time, position and frequency. Consequently, according to the central limit theorem and supposing that the number of averaged probabilistic intensities $M_{b}$ is sufficiently large, the resulting values of probabilistic intensity $I_{b}$ follow a normal distribution. The mean value of this distribution is equal to 0 and it has some standard deviation $\sigma_{b}$. If all the values averaged in the $\operatorname{bin} b$ were independent, the number of independent data points $M_{b}^{\prime}$ in the bin would be equal to the total number of the data points in the bin $M_{b}$ and $\sigma_{b}$ could be calculated as:

$$
\sigma_{b}=\frac{1}{\sqrt{12 M_{b}}}
$$

However, a problem when performing such kind of calculation is that although we know the total number of cumulative probabilities $M_{b}$ included in the bin $b$, we do not know how many of them can be considered as independent; we do not know the value of $M_{b}^{\prime}$. Now we will show how it can be estimated.

[55] As a lower estimate of $M_{b}^{\prime}$ it is reasonable to use the number $N_{b}$ of different half-orbits of the spacecraft that contribute to the bin. This estimation is quite natural, because a duration of a half-orbit is about 35 minutes, which is longer than a typical timescale of intensity changes of electromagnetic waves in the upper ionosphere. Consequently, the data measured in the two different half orbits 
must be independent. However, a problem is that there is more than one data point contributing to the bin $b$ in each of the orbits. In order to solve this, we suppose a linear relation between $M_{b}^{\prime}$ and $N_{b}$ :

$$
M_{b}^{\prime}=\frac{N_{b}}{\alpha^{2}}
$$

where the meaning of the coefficient $\alpha$ is to define which relative fraction of the data coming from the same half-orbit can be considered as independent. Because at least each half-orbit can be considered as independent (see above), $M_{b}^{\prime}$ is larger than or equal to $N_{b}$ and the coefficient $\alpha$ must be a positive number lower than or equal to 1 . Further, we will suppose that, for a given frequency, $\alpha$ is a universal constant that represents a measure of stability of electromagnetic waves in the altitudes of the DEMETER spacecraft. The lower the value of $\alpha$ is, the more variable is the intensity of electromagnetic waves; the value $\alpha=1$ would mean that all the data contributing to the bin from one half-orbit are dependent. Consequently, $\alpha$ is the same for all the bins at a given frequency and its value can be calculated directly from the experimental data.

[56] For a given frequency, we have a set of $Q$ bins corresponding to different times (for our case we have $Q=$ 48 , because we take data from 5 days before to 3 days after the quake with a 4 hours resolution). For each of them we know the values of $I_{b}$ (probabilistic intensity, a number between -0.5 and 0.5 ) and $N_{b}$ (number of different halforbits contributing to the bin). If we knew the coefficient $\alpha$, we could also calculate an estimate of standard deviation $\sigma_{b}$ using the equations (B2) and (B3):

$$
\hat{\sigma}_{b}=\frac{\alpha}{\sqrt{12 N_{b}}} .
$$

Once knowing the standard deviation $\sigma_{b}$ of the probabilistic intensity in the bin $b$, we could calculate the normalized probabilistic intensity $\Upsilon_{b}$ as:

$$
\Upsilon_{b}=\frac{I_{b}}{\sigma_{b}} .
$$

The advantage of this concept is that we know exactly the expected distribution of normalized probabilistic intensities $\Upsilon$ : they should follow the Gaussian distribution with a mean value 0 (the same as probabilistic intensities $I$ ), but their standard deviation should be due to the normalization equal to 1 . Having $Q$ values of the normalized probabilistic intensity, we can therefore write:

$$
\sqrt{\frac{1}{Q} \sum_{b=1}^{Q} \Upsilon_{b}^{2}}=1
$$

which can be rewritten using equations (B4) and (B5) in order to enable the calculation of the coefficient $\alpha$ :

$$
\alpha=\sqrt{\frac{12}{Q} \sum_{b=1}^{Q} N_{b} I_{b}^{2}} .
$$

[57] Using the equation (B7) we can therefore calculate the value of the coefficient $\alpha$ directly from the experimental data. Then, equation (B4) is used to obtain the standard deviation for each of the bins. Finally, normalized probabilistic intensities $\tilde{\mathrm{O}}_{b}$ are calculated according to the equation (B5). These values represent the final results: they express a change of power spectral density of electric field fluctuations as compared to the common natural background and its statistical significance.

[58] Acknowledgments. This work was supported by the Centre National d'Etudes Spatiales (CNES). It is based on observations with the electric field experiment ICE embarked on DEMETER. We thank J. J. Berthelier, PI of the electric field experiment, for the use of the data. We acknowledge the support of the PICS grant 3725 from CNRS/DREI. F. N. and O.S. acknowledge support of the GACR grant 205-06-1267.

[59] Amitava Bhattacharjee thanks Pier Francesco Biagi and another reviewer for their assistance in evaluating this paper.

\section{References}

Asada, T., H. Baba, M. Kawazoe, and M. Sugiura (2001), An attempt to delineate very low frequency electromagnetic signals associated with earthquakes, Earth, Planets Space, 53, 55-62.

Berthelier, J. J., et al. (2006), ICE, the electric field experiment on DEMETER, Planet. Space Sci., 54, 456-471.

Bortnik, J., J. W. Cutler, C. Dunson, and T. E. Bleier (2008), The possible statistical relation of $\mathrm{Pcl}$ pulsations to Earthquake occurrence at low latitudes, Ann. Geophys., 26, 2825-2836.

Clilverd, M. A., C. J. Rodger, and N. R. Thomson (1999), Investigating seismoionospheric effects on a long subionospheric path, J. Geophys. Res., 104(A12), 28,171-28,179.

Freund, F. T. (2007), Pre-earthquake signals: part 1. Deviatoric stresses turn rocks into a source of electric currents, Nat. Hazards Earth Syst. Sci., 7, $535-541$

Gershenzon, N. I., M. B. Gokhberg, A. V. Karakin, N. V. Petviashvili, and A. L. Rykunov (1989), Modelling the connection between earthquake preparation processes and crustal electromagnetic emission, Phys. Earth Planet. Int., 57, 129-138.

Henderson, T. R., V. S. Sonwalkar, R. A. Helliwell, U. S. Inan, and A. C. Fraser-Smith (1993), A search for ELF/VLF emissions induced by earthquakes as observed in the ionosphere by the DE 2 satellite, J. Geophys. Res., 98(A6), 9503-9514.

Hobara, Y., F. Lefeuvre, M. Parrot, and O. A. Molchanov (2005), Low-latitude ionospheric turbulence observed by Aureol-3 satellite, Ann. Geophys., 23, $1259-1270$

Larkina, V. I., V. V. Migulin, O. A. Molchanov, I. P. Kharkov, A. S. Inchin, and V. B. Schvetcova (1989), Some statistical results on very low frequency radiowave emissions in the upper ionosphere over earthquake zones, Phys. Earth Planet. Int., 57, 100-109.

Milne, J. (1890), Earthquakes in connection with electric and magnetic phenomena, Trans. Seismol. Soc. Jpn., 5, 135.

Molchanov, O. A., and M. Hayakawa (1998), On the generation mechanism of ULF seismogenic electromagnetic emissions, Phys. Earth Planet. Int., $105,201-210$.

Molchanov, O. A., O. A. Mazhaeva, A. N. Goliavin, and M. Hayakawa (1993), Observation by the Intercosmos-24 satellite of ELF-VLF electromagnetic emissions associated with earthquakes, Ann. Geophys., 11, $431-440$.

Molchanov, O. A., M. Hayakawa, and V. A. Rafalsky (1995), Penetration characteristics of electromagnetic emissions from an underground seismic source into the atmosphere, ionosphere, and magnetosphere, J. Geophys. Res., 100(A2), 1691-1712.

Molchanov, O. A., A. Kulchitsky, and M. Hayakawa (2001), Inductive seismo-electromagnetic effect in relation to seismogenic ULF emission, Nat. Hazards Earth Syst. Sci., 1, 61-67.

Molchanov, O., et al. (2006), Global diagnostics of the ionospheric perturbations related to the seismic activity using the VLF radio signals collected on the DEMETER satellite, Nat. Hazards Earth Syst. Sci., 6, $745-753$.

Němec, F., O. Santolík, M. Parrot, and J. J. Berthelier (2008), Spacecraft observations of electromagnetic perturbations connected with seismic activity, Geophys. Res. Lett., 35, L05109, doi:10.1029/2007GL032517.

Parrot, M. (1994), Statistical study of ELF/VLF emissions recorded by a low-altitude satellite during seismic events, J. Geophys. Res., 99(A12), $23,339-23,347$. 
Parrot, M. (1995), Electromagnetic noise due to earthquakes, in Handbook of Atmospheric Electrodynamics, vol. 2, chap. 4, edited by H. Volland, pp. 95-116, CRC Press, Boca Raton, Fla.

Parrot, M. (Ed.) (2006), First Results of the DEMETER Micro-Satellite, vol. 54, pp. 411-558, Special Issue of Planet. Space Sci., Elsevier, New York.

Parrot, M., and M. M. Mogilevsky (1989), VLF emissions associated with earthquakes and observed in the ionosphere and the magnetosphere, Phys. Earth Planet. Int., 57, 86-99.

Pulinets, S. A., and K. A. Boyarchuk (2004), Ionospheric Precursors of Earthquakes, 1st ed., Springer, New York.

Pulinets, S. A., A. D. Legen'ka, T. V. Gaivoronskaya, and V. K. Depuey (2003), Main phenomenological features of ionospheric precursors of strong earthquakes, J. Atmos. Sol.-Terr. Phys., 65, 1337-1347.
Rodger, C. J., N. R. Thomson, and R. L. Dowden (1996), A search for ELF/ VLF activity associated with earthquakes using ISIS satellite data, J. Geophys. Res., 101(A6), 13,369-13,378.

Sheskin, D. J. (2000), Handbook of Parametric and Nonparametric Statistical Procedures, 2nd ed., CRC Press, Boca Raton, Fla.

Sorokin, V. M., V. M. Chmyrev, and A. K. Yaschenko (2001), Electrodynamic model of the lower atmosphere and the ionosphere coupling, J. Atmos. Sol.-Terr. Phys., 63, 1681-1691.

Tate, J., and W. Daily (1989), Evidence of electro-seismic phenomena, Phys. Earth Planet. Int., 57, 1-10.

F. Němec and M. Parrot, Laboratoire de Physique et Chimie de l'Environnement et de l'Espace, CNRS, 3A Avenue de la Recherche Scientifique, F-45071 Orléans Cedex 2,France. (frantisek.nemec@gmail.com)

O. Santolík, Institute of Atmospheric Physics, Academy of Sciences of the Czech Republic, Bocni II 1401, 14131 Prague 4, Czech Republic. 